\title{
Effect of Artificial Saliva on the Apatite Structure of Eroded Enamel
}

\author{
Xiaojie Wang, ${ }^{1,2}$ Boriana Mihailova, ${ }^{1}$ Arndt Klocke,, 4 \\ Stefanie Heidrich, ${ }^{1}$ and Ulrich Bismayer ${ }^{1}$ \\ ${ }^{1}$ Department of Earth Sciences, University of Hamburg, Grindelallee 48, 20146 Hamburg, Germany \\ ${ }^{2}$ Department of Preventive, Restorative and Pediatric Dentistry, School of Dental Medicine, University of Bern, Freiburgstrasse 7 , \\ CH-3010 Bern, Switzerland \\ ${ }^{3}$ Division of Orthodontics, Department of Orofacial Sciences, The University of California San Francisco, 707 Parnassus Avenue, \\ San Francisco, CA 94143-0438, USA \\ ${ }^{4}$ Department of Orthodontics, University Medical Center Hamburg-Eppendorf, Martinistraße 52, 20246 Hamburg, Germany
}

Correspondence should be addressed to Boriana Mihailova, boriana.mihailova@uni-hamburg.de

Received 25 March 2011; Accepted 2 May 2011

Academic Editor: Sergio Armenta Estrela

Copyright ( 2011 Xiaojie Wang et al. This is an open access article distributed under the Creative Commons Attribution License, which permits unrestricted use, distribution, and reproduction in any medium, provided the original work is properly cited.

\begin{abstract}
Citric acid-induced changes in the structure of the mineral component of enamel stored in artificial saliva were studied by attenuated total reflectance infrared spectroscopy as well as complementary electron probe microanalysis and scanning electron microscopy. The results indicate that the application of artificial saliva for several hours (the minimum time period proved is $4 \mathrm{~h}$ ) leads to slight, partial recovering of the local structure of eroded enamel apatite. However, artificial saliva surrounding cannot stop the process of loosening and breaking of $\mathrm{P}-\mathrm{O}-\mathrm{Ca}$ atomic linkages in enamel subjected to multiple citric acid treatments. Irreversible changes in the atomic bonding within $700 \mathrm{~nm}$ thick enamel surface layer are observed after three times exposure for 1 min to aqueous solution of citric acid having a $\mathrm{pH}$ value of 2.23, with a 24-hour interval between the individual treatments. The additional treatment with basic fluoride-containing solutions $(1.0 \% \mathrm{NaF})$ did not demonstrate a protective effect on the enamel apatite structure per se.
\end{abstract}

\section{Introduction}

Acidic foods or beverages tend to cause tooth erosion, a chemical dissolution of surface hard tissues resulting from a tooth exposure to a variety of acids $[1,2]$. Salivary flow plays an important role in minimizing enamel erosion by acid attack $[2,3]$. To elucidate how saliva counteracts erosive demineralization, several characteristics of enamel, for example, hardness, surface morphology, mineral loss, and lesion depth, have been investigated [4-7]. It is speculated that saliva buffering capacity, content of calcium and phosphate groups, pellicle acquired on tooth surface and so forth, can act either solely or in combination to inhibit the tooth erosive procedures [8-10]. In vitro and in vivo studies proved that natural saliva and its synthetic substitutes reduce enamel mineral loss $[4,5]$, enhance enamel rehardening $[7,11]$, and decrease erosive lesion depth $[4,5]$. Sodium fluoride solutions have been aimed at minimizing erosive processes.
Recently, it has been reported that the in vitro application of $\mathrm{NaF}$ solutions on enamel reduces the acidic-induced mineral loss [12, 13]. Hughes et al. [14] also found that fluoride application in acidic solutions or as a pretreatment reduced enamel erosion although the benefit observed was small and probably not clinically relevant. Hove et al. $[15,16]$ came to a similar conclusion when investigating enamel exposed to $\mathrm{HCl}$ (simulating a gastric reflux situation); while the in vitro study demonstrated a reduction of etching depth by $25 \%$ due to a protective effect of $\mathrm{NaF}$, a followup in situ study could not confirm the protective effect. Hence, further investigations are required to better understand the effect of saliva and sodium fluoride on the interatomic bonding in the mineral component of eroded enamel.

Recently, attenuated total reflectance infrared (ATR IR) spectroscopy has been successfully applied to analyze the effect of tooth bleaching and acid challenge on enamel crystal structure $[17,18]$. This technique probes the crystal structure 
of outermost layers of the tooth tissue within micron and submicron thickness. It was demonstrated that the position of the most intense ATR IR peak, which arises from the antisymmetrical $v_{3}\left(\mathrm{PO}_{4}\right)$ mode of apatite, indicates the occurrence of structural changes in surficial enamel apatite treated with acidic reagents $[17,18]$. Combined chemical and spectroscopic analyses showed that acidic agents loosen and/or even break a part of the $\mathrm{Ca}-\mathrm{O}$ bonds in apatite, which leads to stiffening of the adjacent P-O bonds and, subsequently, to a shift of the major ATR IR peak to higher wavenumbers [18]. Our previous in vitro study on the local structural alteration of enamel apatite induced by citric acid and NaF-containing solutions revealed that the application of fluoride alone does not suppress the local structural changes in apatite exposed to acidic agents [17].

Saliva is a very important biological parameter related to dental erosion development [19]. Several mechanisms might be involved into the protection of saliva against tooth erosion: (i) saliva directly acts on the erosive agent itself by diluting, clearing, neutralizing, and buffering acids; (ii) the organic components of saliva may form a thin protective film (pellicle) on the enamel surface, which acts as a diffusion barrier or a perm-selective membrane, preventing direct contact between the acids and tooth surface and thus affecting the dissolution rate of dental hard tissue; (iii) due to the content of calcium and phosphate ions, saliva serves as a natural reservoir for apatite regrowth and/or nucleation of new nanocrystals. To elucidate which of these three effects plays a major protective role, the three contributions should be analyzed independently.

The aim of this study is to shed light on the effect of saliva as a source of calcium and phosphate ions as well as to check if the additional application of a basic sodium fluoride solution would influence the effect of saliva. For this purpose, citric acid-induced changes in the atomic structure and bonding of enamel stored in distilled water and in an artificial saliva environment were compared, using ATR IR spectroscopy as well as complementary electron probe microanalysis (EPMA) and scanning electron microscopy (SEM). Additionally, the effect of topical application of sodium fluoride on the eroded enamel stored in artificial saliva was analyzed.

\section{Materials and Methods}

2.1. Experimental Procedure. The enamel specimens used in this study were prepared from noncarious human molars. Slabs parallel to the enamel-dentin junction function (see Figure 1) and of approximate size $3 \times 2 \times 2 \mathrm{~mm}$ were cut from the middle one-third of the enamel surface using a diamond wire saw. The outer surface of the enamel slabs was polished and further analyzed. About $100 \mu \mathrm{m}$ of the outermost enamel layer was removed during the process of grinding and polishing. A suspension of 100-nm-sized colloidal silica was used at the final stage of polishing. The achieved quality of surface smoothness was excellent for spectroscopic, EPMA, and SEM experiments. To avoid any uncertainties and misinterpretation of the experimental data due to tooth-to-tooth variability, adjacent cuts from the

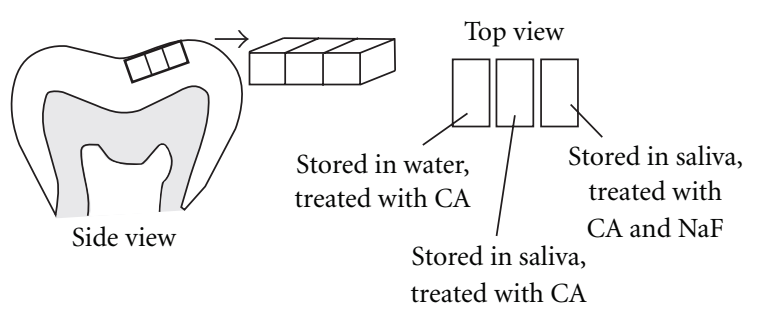

FIGURE 1: Sketch of enamel specimen design.

same tooth were subjected to different types of treatments (see Figure 1). When such an experimental design is used, the quantitative comparison of the effect of the treatment procedure is much more reliable, since ATR IR spectroscopy is sensitive to the specimen orientation as well as to the tooth-to-tooth variation in resistance to acidic challenge, which in turn is related to variations in tooth enamel texture, apatite crystal chemistry, organic-mineral component ratio, and so forth, [17, 18]. Spectra collected from specimens prepared from different teeth show slight statistical variations in the absolute peak positions and relative intensities, but the treatment-induced changes in the spectral features are independent of the specimen origin. We have verified this on more than 10 samples. Hence, for a better comparison, the experimental results presented in the paper are obtained on slabs cut from the same tooth, but the statistics over cuts from different teeth revealed the repeatability of the dependence of the spectral parameters on the sequence of chemical treatment.

In the first version of this paper we have already stated that spectra collected from specimens prepared from different teeth show slight statistical variations in the absolute peak positions and relative intensities, but the treatmentinduced changes in the spectral features are independent of the specimen origin. We have verified this on more than 10 samples. However, we present the data collected only on one.

We applied three different treatment procedures to adjacent cuts of the same tooth.

(A) Enamel specimens were stored in distilled water at $37^{\circ} \mathrm{C}$ and manifold treated with a $0.1 \mathrm{~mol} / \mathrm{L}$ citric acid solution having a $\mathrm{pH}$ value of 2.23; the time of a single acidic exposure was $1 \mathrm{~min}$.

(B) Enamel specimens were firstly immersed into artificial saliva at $37^{\circ} \mathrm{C}$ for 7 days and then submitted to a deremineralization cycle repeated three times. The demineralization treatment was performed also in a $0.1 \mathrm{~mol} / \mathrm{L}$ citric acid solution for 1-minute. The short period of a $1 \mathrm{~min}$ exposure was chosen to mimic the conditions during beverage consumption, while the remineralization treatment was carried out in artificial saliva at $37^{\circ} \mathrm{C}$ for at least $4 \mathrm{~h}$. After each treatment with citric acid solution, the samples were rinsed with distilled water, dried in air, and subjected to ATR spectroscopic measurements, while after each period of storage in artificial saliva, the samples were only dried in air and then subjected to ATR spectroscopic measurements. The artificial saliva was changed every $24 \mathrm{~h}$.

(C) The same treatment as in procedure $\mathrm{B}$, but the samples were additionally exposed to a $1.0 \% \mathrm{NaF}$ solution 
with a $\mathrm{pH}$ value of 10.05 for $5 \mathrm{~min}$ three times daily. This regimen was chosen to simulate a clinical situation using topical fluoride therapy recommended to prevent erosion.

Thus, the comparison between enamel subjected to procedure $\mathrm{A}$ and that subjected to procedures $\mathrm{B}$ and $\mathrm{C}$ can reveal the effect of artificial saliva on demineralization processes, while the comparison between enamel treated according to procedure $\mathrm{B}$ and procedure $\mathrm{C}$ demonstrates the cooperative effect of saliva and fluoride.

The artificial saliva was prepared according to Klimek et al. [20]: $0.002 \mathrm{~g}$ ascorbic acid, $0.030 \mathrm{~g}$ glucose, $0.580 \mathrm{~g}$ $\mathrm{NaCl}, 0.170 \mathrm{~g} \mathrm{CaCl}_{2}, 0.160 \mathrm{~g} \mathrm{NH}_{4} \mathrm{Cl}, 1.270 \mathrm{~g} \mathrm{KCl}, 0.160 \mathrm{~g}$ NaSCN, $0.330 \mathrm{~g} \mathrm{KH}_{2} \mathrm{PO}_{4}, 0.200 \mathrm{~g}$ urea, $0.340 \mathrm{~g} \mathrm{Na} 2 \mathrm{HPO}_{4}$, and $2.700 \mathrm{~g}$ mucin (Bacto-Mucin Bacteriological) in $1000 \mathrm{~mL}$ distilled water. The solution was further titrated with a phosphate buffer of $26.4 \mathrm{~mL} 0.06 \mathrm{M} \mathrm{Na}_{2} \mathrm{HPO}_{4} 2 \mathrm{H}_{2} \mathrm{O}$ and $7.36 \mathrm{~mL} 0.06 \mathrm{M} \mathrm{KH}_{2} \mathrm{PO}_{4}$, following the procedure described by Burgmaier et al. [21]. The achieved $\mathrm{pH}$ value of the artificial saliva solution was 8.0. This $\mathrm{pH}$ value is representative of saliva under conditions of stimulation [22] and is, thus, relevant for remineralization processes.

Aqueous solutions of $0.1 \mathrm{~mol} / \mathrm{L}$ citric acid and $1.0 \% \mathrm{NaF}$ were prepared by dissolving an appropriate amount of powder in distilled water.

\subsection{Analytical Methods}

2.2.1. ATR IR. ATR IR spectra were collected with a Bruker Equinox 55 FTIR spectrometer equipped with a Pike MIRacle ATR accessory, having a single-reflection sampling plate and a round contact surface between the ATR crystal and the sample with a diameter of $1.8 \mathrm{~mm}$. The force applied to the sample to achieve a good contact with the ATR crystal was controlled by a micrometer pressure clamp, and for all samples, the magnitude of the force was the same as the maximal allowed by the device. The background spectrum was collected from an optically homogeneous polished plateshaped $\mathrm{KBr}$ crystals ( $\mathrm{KBr}$ window, Korth Kristalle Gmbh). The use of a Ge ATR crystal assured a characteristic penetration depth of approximately $700 \mathrm{~nm}$ at $1000 \mathrm{~cm}^{-1}$, which allowed the separation of the surface IR absorption from the bulk one by enhancing the signal coming from the submicron surface layer. The spectra were recorded with an instrumental resolution of $4 \mathrm{~cm}^{-1}$ resolution, averaging over 512 scans. The spectra were measured in the spectral range $570-4000 \mathrm{~cm}^{-1}$ and subsequently normalized to a constant penetration depth. Only slabs free of cracks observable under an optical microscope were investigated. The repeatability of the spectra was verified by collecting at least two spectra from the same specimen. No variations in the peak positions and relative intensities depending on the positioning of the sample in the ATR unit were detected. Since the contact surface area between the sample and ATR crystal is approximately $2.54 \mathrm{~mm}^{2}$, lateral inhomogeneities of the sample on the micrometer- or submicrometer scale, cannot influence the overall IR signal.

2.2.2. SEM. To observe the surface morphology of dental enamel, samples coated with gold were studied by SEM imaging. The micrographs were recorded with a Zeiss LEO $1455 \mathrm{VP}$ scanning electron microscope operating at $20 \mathrm{kV}$.

2.2.3. EPMA. The lateral chemical inhomogeneity of dental enamel was analyzed by conducting mapping of $\mathrm{Ca}, \mathrm{P}$, $\mathrm{Na}$, and $\mathrm{Cl}$, using an electron probe microanalyzer Cameca Microbeam SX100 equipped with a wavelength- and energydispersive system. The mapping was performed with a step size of $0.1 \mu \mathrm{m}$ and a penetration depth of approximately $1.0 \mu \mathrm{m}$.

2.2.4. Background Results. Previous studies demonstrated that the spectral range of $900-1200 \mathrm{~cm}^{-1}$, which is dominated by the stretching modes of $\mathrm{PO}_{4}$ tetrahedra, is the range most pronouncedly impacted by acid challenge $[17,18]$. ATR IR spectroscopy probes the atomic bonding of the outermost enamel apatite within a layer corresponding to the characteristic penetration depth, which in our experiment is $700 \mathrm{~nm}$. A higher wavenumber of the peak related to the antisymmetric stretching mode of $\mathrm{PO}_{4}$ tetrahedra is indicative of changes in the local structure of hydroxyapatite, namely, occurrence of loosened and/or broken $\mathrm{Ca}-\mathrm{O}$ bonds and thus disturbing the typical atomic surroundings of $\mathrm{PO}_{4}$ groups. Therefore, the degree of local atomic structural damage in surficial enamel apatite can be quantified by the change in the position of the strongest ATR IR peak near $1015 \mathrm{~cm}^{-1}$ with respect to that of untreated enamel.

\section{Results}

Figure 2(a) shows the change in the major peak position of enamel stored in water as a function of the number of treatments with citric acid solution of $\mathrm{pH}=2.23$ for $1 \mathrm{~min}$. The peak positions were determined by fitting the spectral profile in the range of $900-1200 \mathrm{~cm}^{-1}$ using four Lorentz functions (see Figure 2(b)). The number of Lorentzians was consistent with the four internal stretching modes of $\mathrm{PO}_{4}$ tetrahedra: one symmetric and three antisymmetric, which are nondegenerate in the case of irregular tetrahedron. According to group-theory analysis of apatite, the number of crystal phonon modes related to the internal stretching modes is larger than four, due to the multiplicity of the tetrahedral ions in the crystal. However, the experimental IR profile of untreated apatite shows four signals in the range of $900-1200 \mathrm{~cm}^{-1}$, which indicates that the wavenumbers of the crystal phonon modes related to $\mathrm{P}-\mathrm{O}$ stretching are grouped around the wavenumbers of the corresponding internal tetrahedral modes. Hence, the simplest model to fit the spectral range of $900-1200 \mathrm{~cm}^{-1}$ of apatite involves four Lorentzians.

As can be seen in Figure 2(a), after three times of acid exposure, the degree of damage within a surface layer of $\sim 700 \mathrm{~nm}$, which is the characteristic penetration depth of our ATR IR experiments, reaches a saturation level. A similar trend was observed for enamel treated with milder acidic agents for longer time [17]. The statistics over a large number of samples show that when the structural damage of the surface layers reaches a saturation, the change in the position of the major peak is $\sim 15 \mathrm{~cm}^{-1}$ with a deviation of $\pm 4 \mathrm{~cm}^{-1}$. 


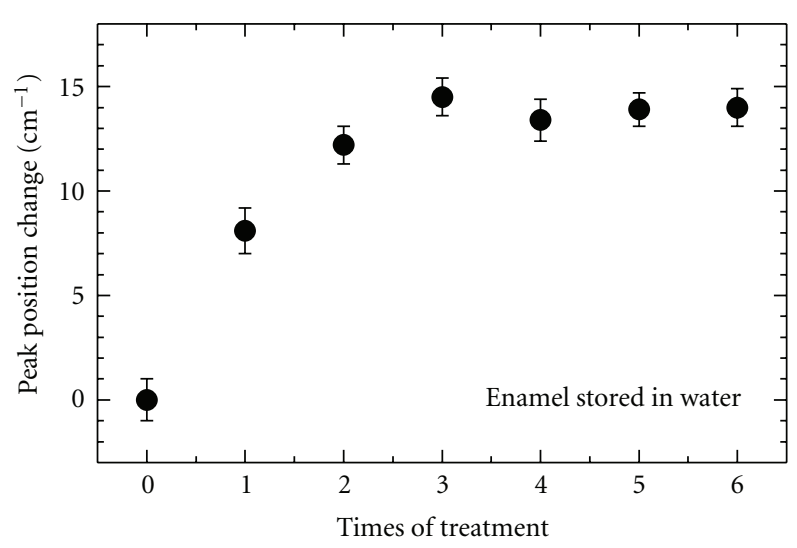

(a)

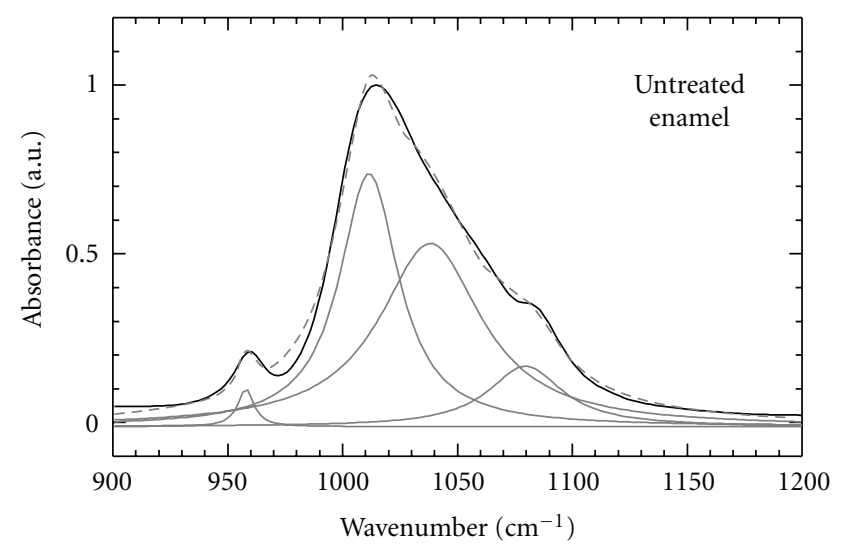

(b)

FIGURE 2: Shift of the major ATR IR peak near $1015 \mathrm{~cm}^{-1}$ for enamel stored in water versus number of 1-minute treatments with citric acid, $\mathrm{pH}=2.23$ (a); experimental spectrum of untreated enamel (bold black line) fitted with 4 Lorentzians (thin gray lines) and the resultant profile (dashed gray line) (b). The error bars in (a) correspond to uncertainties in peak positions resulting from spectrum profile fittings. The position of the second Lorentzian is $1012 \mathrm{~cm}^{-1}$, which value was taken as initial wavenumber to calculate the peak position changes.

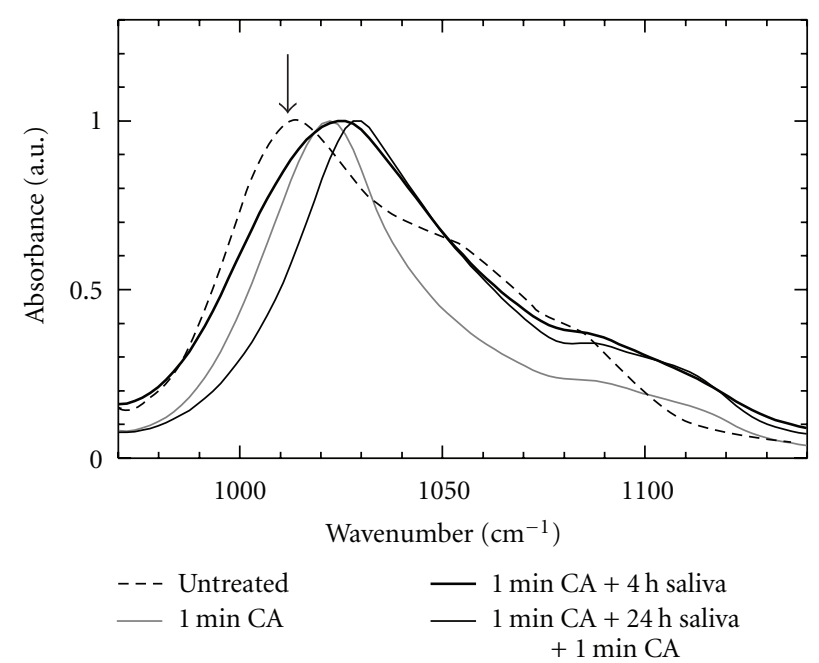

(a)

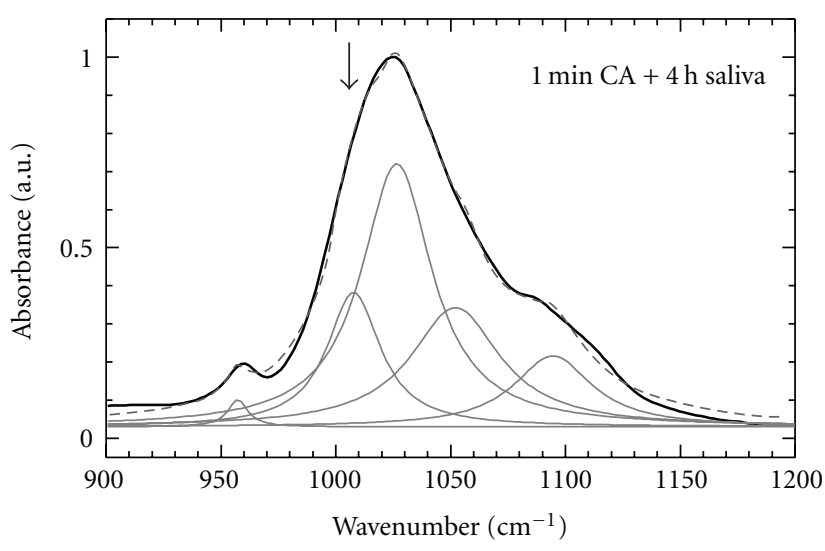

(b)

FIGURE 3: ATR IR spectra of untreated enamel (dashed line), treated with citric acid (CA) for 1 min (thin gray line), treated with CA for $1 \mathrm{~min}$ and then stored in saliva (S) for $4 \mathrm{~h}$ (bold black line), and treated with CA for $1 \mathrm{~min}$, then stored in saliva for $24 \mathrm{~h}$, and treated again with CA for $1 \mathrm{~min}$ (thin black line) (a); experimental spectrum of enamel treated with CA for 1 min and then stored in saliva for $4 \mathrm{~h}$ (bold black line) fitted with 5 Lorentzians (thin gray lines) and the resultant profile (dashed gray line) (b). The arrow in both plots points to the lower-wavenumber shoulder observed for enamel subsequently stored in saliva for $4 \mathrm{~h}$, the position of which matches the position of the major ATR IR peak for untreated enamel.

Further, we compared the effect of citric acid on enamel stored in water and in artificial saliva. The preliminary soaking of enamel in saliva for 7 days did not affect the ATR IR spectrum, which confirms that the used chemical composition of artificial saliva is adequate to probe the acidinduced alteration of apatite atomic bonding in salivary surroundings. After a single treatment with citric acid with $\mathrm{pH}=2.23$ for $1 \mathrm{~min}$, enamel prestored in saliva showed the same ATR IR spectrum as that of enamel kept in water, which indicates that artificial saliva had no protective effect against such an acidic challenge under the experimental conditions. However, subsequent storage of enamel in saliva for $4 \mathrm{~h}$ led to detectable spectral changes. The main peak becomes broader and asymmetric in shape due to the appearance of a weak shoulder positioned lower wavenumbers (see Figure 3). Similar spectral changes were detected at the first step of acidic treatment of enamel subjected to a multistep acidic challenge and it indicates the development of an intermediate 


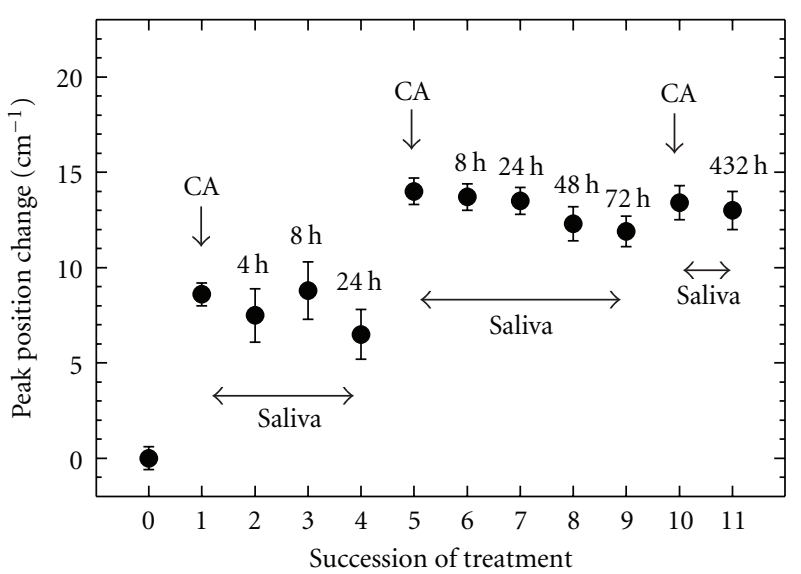

(a)

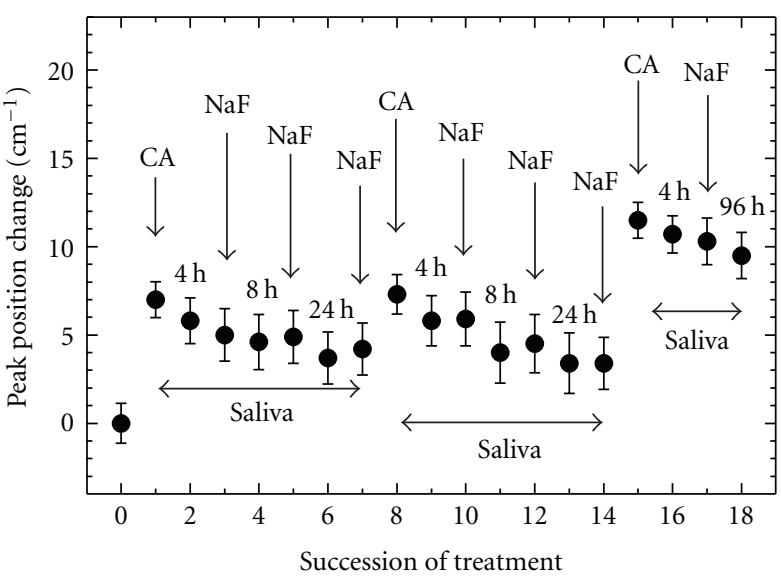

(b)

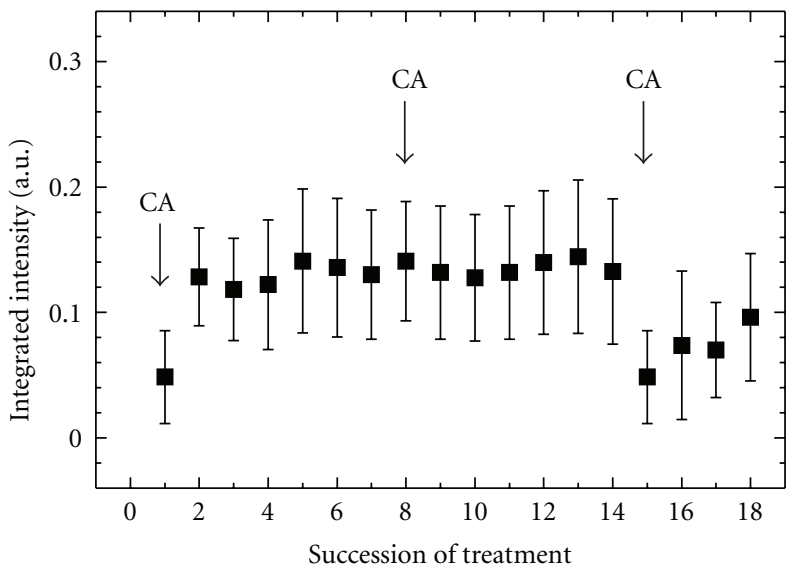

(c)

FIGURE 4: Shift of the major ATR IR peak near $1015 \mathrm{~cm}^{-1}$ for enamel stored in saliva (a) and for enamel stored in saliva and additionally exposed to $\mathrm{NaF}$ (b); each treatment with citric acid (CA) was performed for $1 \mathrm{~min}$; the time of enamel storage in saliva is given with respect to the last treatment with citric acid; each treatment with $\mathrm{NaF}$ was performed for $5 \mathrm{~min}$. The peak positions in (a) and (b) were obtained using a four-Lorentzian model to fit the spectrum profiles between 900 and $1200 \mathrm{~cm}^{-1}$. (c) displays the integrated intensity of the lowerwavenumber side signal (see Figure 3) normalized to the total absorption intensity between 900 and $1200 \mathrm{~cm}^{-1}$, when a five-Lorentzian model was applied to fit the spectrum profiles between 900 and $1200 \mathrm{~cm}^{-1}$ of samples stored in saliva and additionally exposed to NaF; for simplicity, only the points representing the citric-acid treatment are marked in (c); all other points exactly correspond to the points in (b).

structural stage consisting of spatial regions of heavily damaged apatite within a matrix of almost undamaged apatite [17]. Therefore, the results obtained on enamel stored in saliva after acidic exposure demonstrate a recovery effect of saliva on the local atomic structure of enamel apatite.

The signals arising from the affected and nonaffected/recovered regions are very close in wavenumber and overlap. Therefore, the two signals can be modelled as one Lorentzian, whose wavenumber would represent the weight coefficient of the two signals: a higher wavenumber indicates a larger contribution of the signal from the damaged regions, while a lower wavenumber indicates a larger contribution form the undamaged regions. Thus, when a four-Lorentzian model is used to fit the spectra of untreated and treated samples, the change in the peak position of the most intense IR band near $1015 \mathrm{~cm}^{-1}$ reflects the change in the fraction of damaged regions in enamel. Additionally, we have applied a five-Lorentzian model to the spectra of samples stored in saliva. In this case, we have modelled the contribution from damaged and undamaged/recovered areas to the most intense IR band with two separate replicas. Thus, the trend in the intensity of the lower-wavenumber side band (see Figure 3) normalized to the total intensity in the range $900-1200 \mathrm{~cm}^{-1}$ represents the structural state of superficial enamel subjected to a deremineralization process: a lower intensity corresponds to a high degree of structural damage, while an increase in the intensity indicates recovering of the apatite structure. However, due to the strong overlapping of the peaks, the uncertainties in determining peak integrated intensities within a five-Lorentzian model are relatively large, which hampers the precise quantification of the degree structural damage.

Figure 4 presents the trends of the spectral features of enamel stored in saliva. The change in the peak position as 


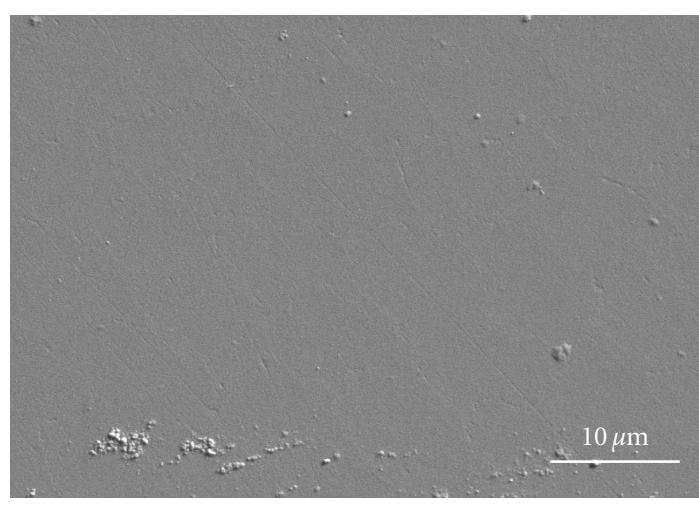

(a)

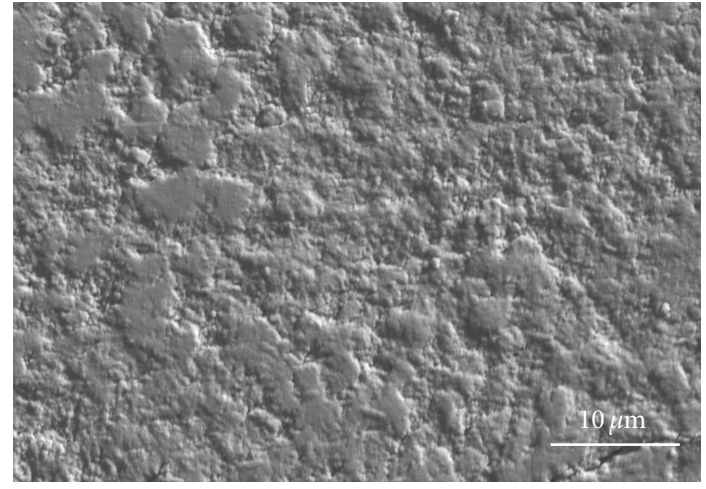

(b)

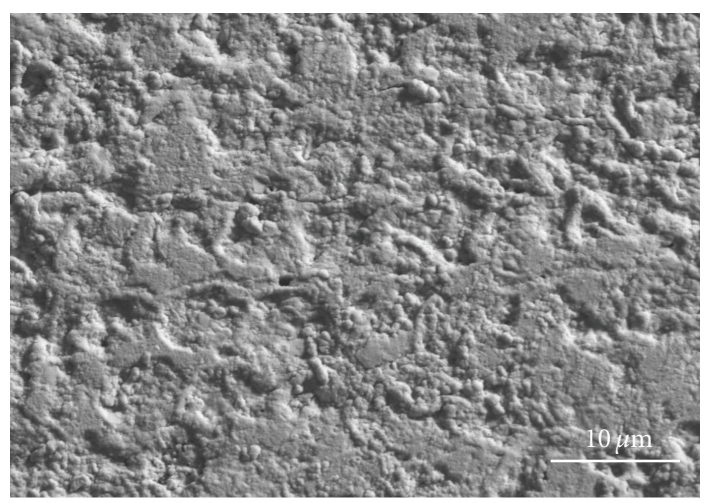

(c)

FIGURE 5: SEM micrographs of untreated enamel (a); enamel treated with citric acid and then remineralized in artificial saliva (b); enamel treated with citric acid and then remineralized in artificial saliva in combination with additional treated with $\mathrm{NaF}(\mathrm{c})$.

determined within a four-Lorentz model for enamel treated according to procedure $\mathrm{B}$ and $\mathrm{C}$ stored is shown in Figures $4(\mathrm{a})$ and 4(b), respectively. It is seen that artificial salivary environment leads to a partial recovery of the mineral component of enamel, but it cannot stop the overall violation of the atomic bonding in apatite upon multiple acidic attack.

The comparison between ATR IR data on enamel stored in saliva without and with additional $\mathrm{NaF}$ treatment (see Figures 4(a) and 4(b), resp.) reveals that topical application of $\mathrm{NaF}$ to artificial saliva does not inhibit the long-term structural alteration in eroded enamel. After a third acid exposure, the peak position change for enamel stored in saliva and additionally treated with $\mathrm{NaF}$ became the same as that of the peak position change for enamel stored in saliva, without being additionally treated with $\mathrm{NaF}$. The use of the relative intensity of the lower-wavenumber side band (determined within the five-Lorentzian model) to estimate the structural state (see Figure 4(c)) confirms the results obtained within the four-Lorentzian model: the combination of saliva and a repetitive treatment with $\mathrm{NaF}$ opposes to the impact of citric acid after two times acidic exposure but have only a diminutive effect after a third treatment with citric acid.

SEM micrographs also show no detectible effect of $\mathrm{NaF}$ treatment in the experiment (Figure 5). The morphological changes observed for enamel subjected to a combination of artificial saliva and $\mathrm{NaF}$ resemble those observed for enamel kept in artificial saliva, demonstrating in both cases apparent etching-like pattern. Moreover, the comparison between the $\mathrm{Ca}$ - and $\mathrm{P}$-mapping images of enamel exposed to saliva and to saliva $+\mathrm{NaF}$ reveals a similar degree of lateral chemical inhomogeneity in the eroded surface (Figure 6). The mapping of $\mathrm{Na}$ and $\mathrm{Cl}$ (not shown) also revealed no difference between samples treated with artificial saliva alone and in combination with $\mathrm{NaF}$.

\section{Discussion}

The position and the shape of the major ATR IR peak is a result of the existence of two overlapping components corresponding to noneroded and eroded enamel apatite. Figure 7 schematically represents the proposed mechanism of structure transformation processes in superficial enamel exposed first to citric acid and then to saliva. Initially, the treatment with citric acid leads to a formation of spatial areas with damaged apatite that exhibits local structural alteration, namely, broken and/or loosened P-O-Ca atomic linkages. After a prolonged treatment with citric acid, the entire surface layer probed by ATR IR spectroscopy (approximately $700 \mathrm{~nm}$ in thickness) is affected, and it consists of apatite with a high degree of local structural defects: $\mathrm{PO}_{4}$ species 

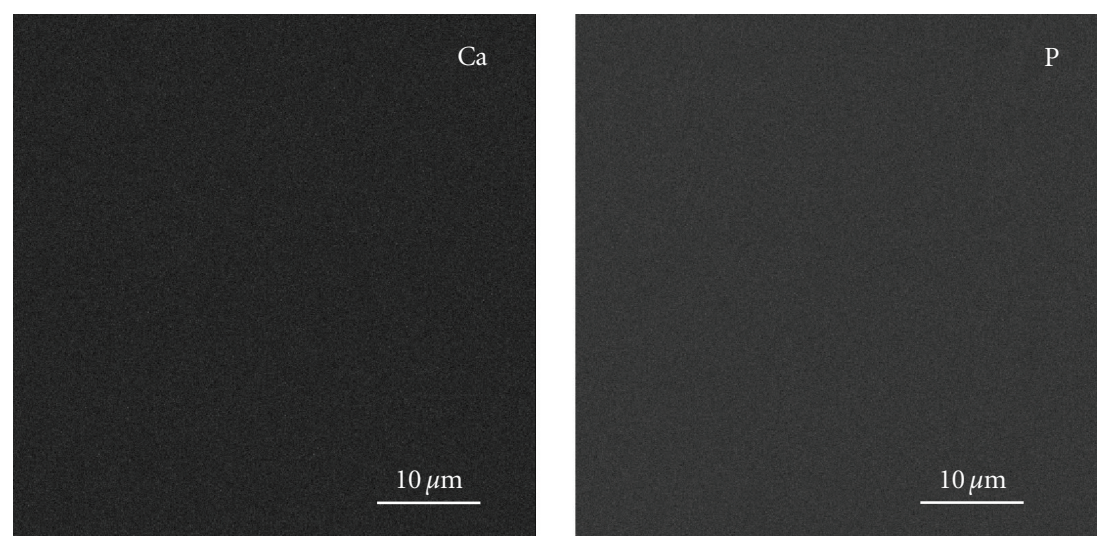

(a)
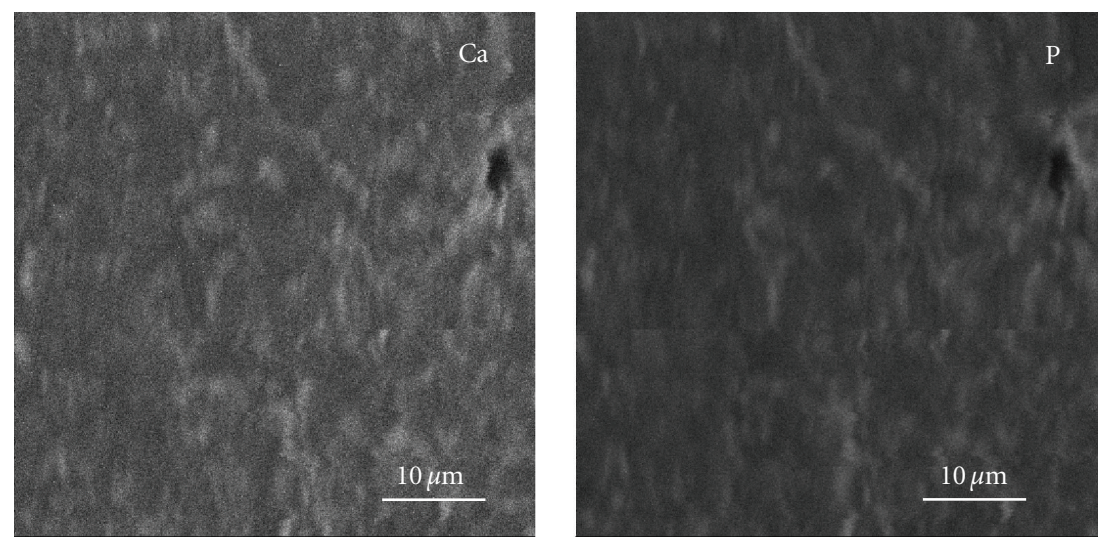

(b)
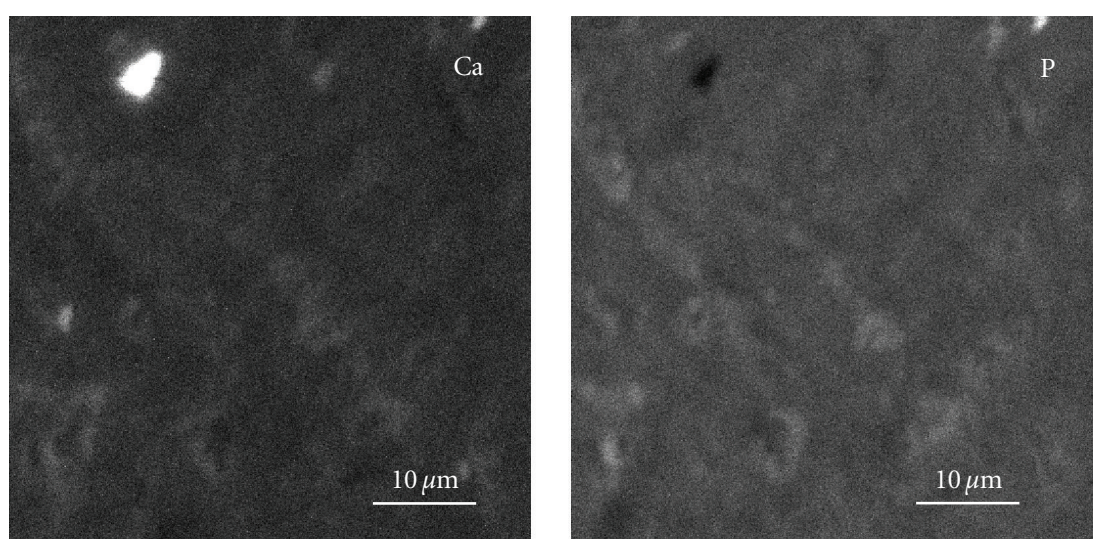

(c)

Figure 6: Electron-probe P- of Ca-mapping of untreated enamel (a); enamel treated with citric acid and then remineralized in artificial saliva (b); enamel treated with citric acid and then remineralized in artificial saliva in combination with additional treated with $\mathrm{NaF}$ (c).

with stiffer $\mathrm{P}-\mathrm{O}$ bonds due to break and/or loosening of adjacent $\mathrm{Ca}-\mathrm{O}$ bonds. Saliva stabilizes atomic linkages in apatite and favours the reappearance of spatial areas of defect-poor apatite. The resultant ATR IR peak shift with respect to position of the peak of untreated enamel actually reflects the different contribution of the two overlapping components: noneroded and eroded apatite. More abundant eroded enamel regions correspond to a larger peak shift.
Hence, the change in the major peak position is a reliable indicator of the degree of structural damage of apatite within a $700 \mathrm{~nm}$ surface layer. The spectroscopic results reveal subtle structural recovery of enamel eroded by citric acid with $\mathrm{pH}=$ 2.23 for $1 \mathrm{~min}$ after storage in artificial saliva for 4 hours. The effect of remineralization by saliva is more pronounced after $24 \mathrm{~h}$; however, prolonged time of 3 days (see Figure 5(a)) does not lead to any further substantial changes. A repeated 
Partial damage of the surface layer (apatite with broken/loose $\mathrm{P}-\mathrm{O}-\mathrm{Ca}$ linkages) due to citric acid

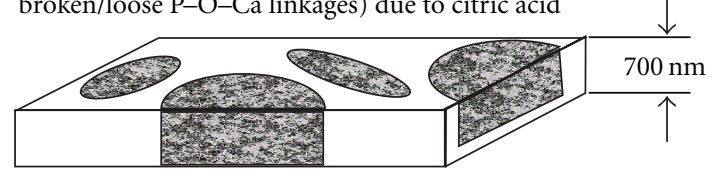

Complete damage of the $700 \mathrm{~nm}$ layer (apatite with altered $\mathrm{P}-\mathrm{O}-\mathrm{Ca}$ linkages) after prolonged acidic challenge

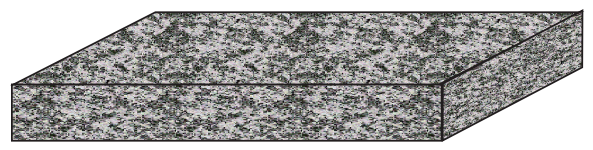

Partial recovery of atomic linkages in apatite due to saliva

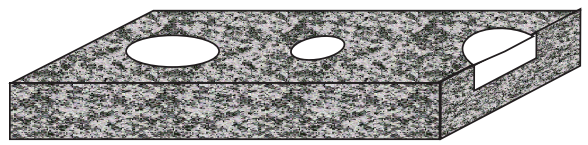

FIGURE 7: Sketch of deremineralization processes on enamel mineral surface layer of thickness $\sim 700 \mathrm{~nm}$; areas of undamaged apatite and damaged apatite (with broken or loose $\mathrm{P}-\mathrm{O}-\mathrm{Ca}$ atomic linkages) are represented by white and textured gray color, respectively.

application of deremineralization cycle leads to a more severe damage of enamel apatite, which cannot be recovered even after an exposure to artificial saliva for several days. These results, which are based on the change in atomic bonding and local arrangements, explain the detrimental effect of frequent intake of acidic foods and beverages, as resulting from the imbalance between acidic demineralization and salivary remineralization, which consequently leads to irreversible lesions of enamel apatite.

The results of the present study show that the topical application of $\mathrm{NaF}$ in the presence of artificial saliva does not suppress or reverse the loosening and break of atomic P-OCa linkages of enamel repeatedly exposed to acidic challenge. This indicates that saliva as a chemical reservoir is not the major reason for the enhancement of the long-term enamel resistance to an acidic challenge under a topical application of fluoride. Therefore, the mechanism of saliva + fluoride protective effect should be related to the $\mathrm{pH}$ value and/or the organic components of saliva. This is in agreement with previous findings based on in vitro study of enamel treated with various F-containing solutions [17], demonstrating that the potential of fluorine-containing agents to prevent enamel apatite from undesired acid-induced changes should be related mostly to the formation of $\mathrm{CaF}_{2}$ protective layer, which in turn depends on the $\mathrm{pH}$ value of the medium surrounding the enamel surface. Several studies on the hardness of enamel in a saliva environment also demonstrated that saliva is incapable of totally recovering the original surface structure of sound enamel [4, 23]. On the other hand, Eisenburger et al. [6] found that upon 24-hour remineralization in artificial saliva, eroded enamel returns to its normal level of resistance to ultrasonication. The controversial conclusions from different studies underline the complex effect of saliva and the necessity of well-designed experiments in order to check the primary reason for the inhibition of the acidinduced damage in natural saliva environment.

The artificial saliva utilized in the present study was aimed at simulating saliva relevant for remineralization processes. Therefore, the $\mathrm{pH}$ value of the saliva was adjusted to levels natural saliva reaches under stimulation conditions and, thus, demonstrates improved salivary buffer capacity [22]. However, it should be emphasized that artificial saliva cannot model demineralization and remineralization processes in the intraoral environment related to various biological factors, such as salivary pellicle and biofilm, as well as to inherent $\mathrm{F}$ that favours the formation of protective $\mathrm{CaF}_{2}$ layer. In general, natural saliva might offer a greater protection than artificial saliva due to the ability to form salivary pellicle that restricts the acid diffusion and transport of ions in and out of the enamel surface [24-27]. Therefore, under clinical conditions, the demineralization due to an erosive attack may be less pronounced compared to the conditions used in the current study.

In conclusion, partial recovery of the atomic bonding in the eroded enamel apatite occurs after several hours due to the favourable influence of the surrounding saliva. However, saliva as a chemical reservoir of calcium and phosphate ions cannot prevent irreversible local structural changes within a $700 \mathrm{~nm}$ layer of enamel apatite repeatedly exposed to acidic agents. Topical treatment with basic fluoride-containing agents seems to show a diminutive contribution to the ability of artificial saliva, supplying calcium and phosphate ions, to oppose to the acid-induced violation of the local atomic structure of enamel apatite.

\section{Acknowledgments}

The authors would like to thank Peter Stutz (University of Hamburg) for cutting and polishing dental samples and Yvonne Milker (University of Hamburg) for conducting SEM experiments. Financial support by the Deutsche Forschungsgemeinschaft (GRK-611) is gratefully acknowledged.

\section{References}

[1] J. M. ten Cate and T. Imfeld, "Dental erosion, summary," European Journal of Oral Sciences, vol. 104, no. 2, pp. 240-244, 1996.

[2] A. Lussi, T. Jaeggi, and D. Zero, "The role of diet in the aetiology of dental erosion," Caries Research, vol. 38, supplement 1, pp. 34-44, 2004.

[3] G. A. Sánchez and M. V. Fernandez De Preliasco, "Salivary pH changes during soft drinks consumption in children," International Journal of Paediatric Dentistry, vol. 13, no. 4, pp. 251-257, 2003.

[4] B. T. Amaechi and S. M. Higham, "In vitro remineralisation of eroded enamel lesions by saliva," Journal of Dentistry, vol. 29, no. 5, pp. 371-376, 2001.

[5] B. T. Amaechi and S. M. Higham, "Eroded enamel lesion remineralization by saliva as a possible factor in the sitespecificity of human dental erosion," Archives of Oral Biology, vol. 46, no. 8, pp. 697-703, 2001.

[6] M. Eisenburger, J. Hughes, N. X. West, R. P. Shellis, and M. Addy, "The use of ultrasonication to study remineralisation of eroded enamel," Caries Research, vol. 35, no. 1, pp. 61-66, 2001. 
[7] D. Rios, H. M. Honório, A. C. Magalhães et al., "Effect of salivary stimulation on erosion of human and bovine enamel subjected or not to subsequent abrasion: an in situ/ex vivo study," Caries Research, vol. 40, no. 3, pp. 218-223, 2006.

[8] A. F. Hall, C. A. Buchanan, D. T. Millett, S. L. Creanor, R. Strang, and R. H. Foye, "The effect of saliva on enamel and dentine erosion," Journal of Dentistry, vol. 27, no. 5, pp. 333339, 1999.

[9] T. Jaeggi and A. Lussi, "Toothbrush abrasion of erosively altered enamel after intraoral exposure to saliva: an in situ study," Caries Research, vol. 33, no. 6, pp. 455-461, 1999.

[10] T. Attin, S. Knöfel, W. Buchalla, and R. Tütüncü, "In situ evaluation of different remineralization periods to decrease brushing abrasion of demineralized enamel," Caries Research, vol. 35, no. 3, pp. 216-222, 2001.

[11] Y. Nekrashevych and L. Stösser, "Protective influence of experimentally formed salivary pellicle on enamel erosion: an in vitro study," Caries Research, vol. 37, no. 3, pp. 225-231, 2003.

[12] N. Schlueter, C. Ganss, U. Mueller, and J. Klimek, "Effect of titanium tetrafluoride and sodium fluoride on erosion progression in enamel and dentine in vitro," Caries Research, vol. 41, no. 2, pp. 141-145, 2007.

[13] C. Ganss, N. Schlueter, M. Hardt, P. Schattenberg, and J. Klimek, "Effect of fluoride compounds on enamel erosion in vitro: a comparison of amine, sodium and stannous fluoride," Caries Research, vol. 42, no. 1, pp. 2-7, 2008.

[14] J. A. Hughes, N. X. West, and M. Addy, "The protective effect of fluoride treatments against enamel erosion in vitro," Journal of Oral Rehabilitation, vol. 31, no. 4, pp. 357-363, 2004.

[15] L. Hove, B. Holme, A. Young, and A. B. Tveit, "The protective effect of $\mathrm{TiF}_{4}, \mathrm{SnF}_{2}$ and $\mathrm{NaF}$ against erosion-like lesions in situ," Caries Research, vol. 42, no. 1, pp. 68-72, 2008.

[16] L. Hove, B. Holme, B. Øgaard, T. Willumsen, and A. B. Tveit, "The protective effect of $\mathrm{TiF}_{4}, \mathrm{SnF}_{2}$ and $\mathrm{NaF}$ on erosion of enamel by hydrochloric acid in vitro measured by white light interferometry," Caries Research, vol. 40, no. 5, pp. 440-443, 2006.

[17] X. Wang, A. Klocke, B. Mihailova, L. Tosheva, and U. Bismayer, "New insights into structural alteration of enamel apatite induced by citric acid and sodium fluoride solutions," Journal of Physical Chemistry B, vol. 112, no. 29, pp. 8840-8848, 2008.

[18] X. Wang, B. Mihailova, A. Klocke et al., "Side effects of a non-peroxide-based home bleaching agent on dental enamel," Journal of Biomedical Materials Research A, vol. 88, no. 1, pp. 195-204, 2009.

[19] A. T. Hara, A. Lussi, and D. T. Zero, "Biological factors," in Dental Erosion: From Diagnosis to Therapy (Monographs in Oral Science), A. Lussi, Ed., vol. 20, pp. 88-99, S. Karger AG, Basel, Switzerland, 2006.

[20] J. Klimek, E. Hellwig, and G. Ahrens, "Fluoride taken up by plaque, by the underlying enamel and by clean enamel from three fluoride compounds in vitro," Caries Research, vol. 16, no. 2, pp. 156-161, 1982.

[21] G. M. Burgmaier, I. M. Schulze, and T. Attin, "Fluoride uptake and development of artificial erosions in bleached and fluoridated enamel in vitro," Journal of Oral Rehabilitation, vol. 29, no. 9, pp. 799-804, 2002.

[22] R. A. D. Williams and J. C. Elliott, Basic and Applied Dental Biochemistry, Churchill Livingstone, Edinburgh, Scotland, 1989.

[23] H. Devlin, M. A. Bassiouny, and D. Boston, "Hardness of enamel exposed to Coca-Cola $(\mathrm{R}$ ) and artificial saliva," Journal of Oral Rehabilitation, vol. 33, no. 1, pp. 26-30, 2006.
[24] R. T. Zahradnik, D. Propas, and E. C. Moreno, "In vitro enamel demineralization by Streptococcus mutans in the presence of salivary pellicles," Journal of Dental Research, vol. 56, no. 9, pp. 1107-1110, 1977.

[25] B. L. Slomiany, V. L. N. Murty, I. D. Mandel, S. Sengupta, and A. Slomiany, "Effect of lipids on the lactic acid retardation capacity of tooth enamel and cementum pellicles formed in vitro from saliva of caries-resistant and caries-susceptible human adults," Archives of Oral Biology, vol. 35, no. 3, pp. 175180, 1990.

[26] B. T. Amaechi, S. M. Higham, W. M. Edgar, and A. Milosevic, "Thickness of acquired salivary pellicle as a determinant of the sites of dental erosion," Journal of Dental Research, vol. 78, no. 12, pp. 1821-1828, 1999.

[27] M. Hannig and M. Balz, "Influence of in vivo formed salivary pellicle on enamel erosion," Caries Research, vol. 33, no. 5, pp. 372-379, 1999. 


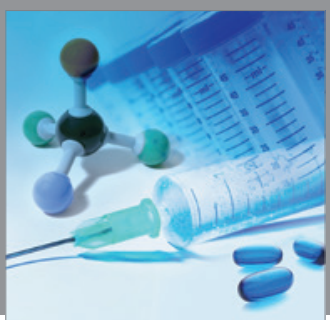

International Journal of

Medicinal Chemistry

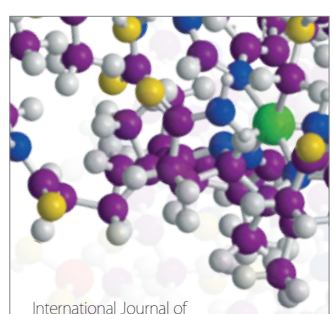

Carbohydrate Chemistry

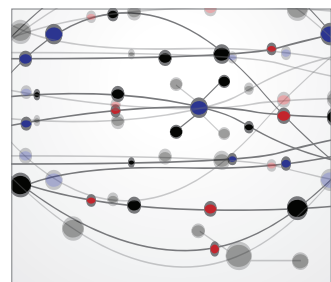

The Scientific World Journal
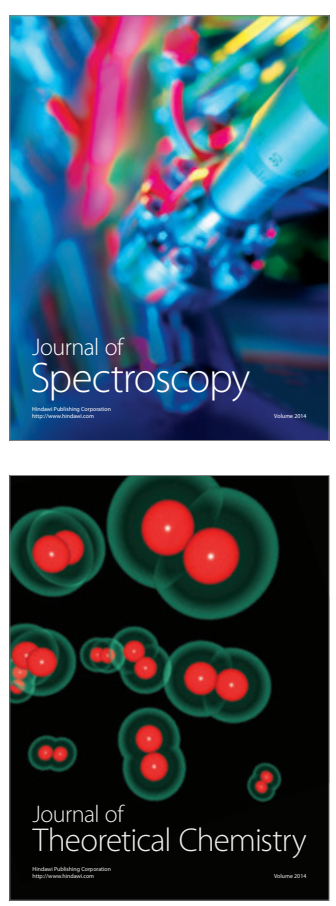
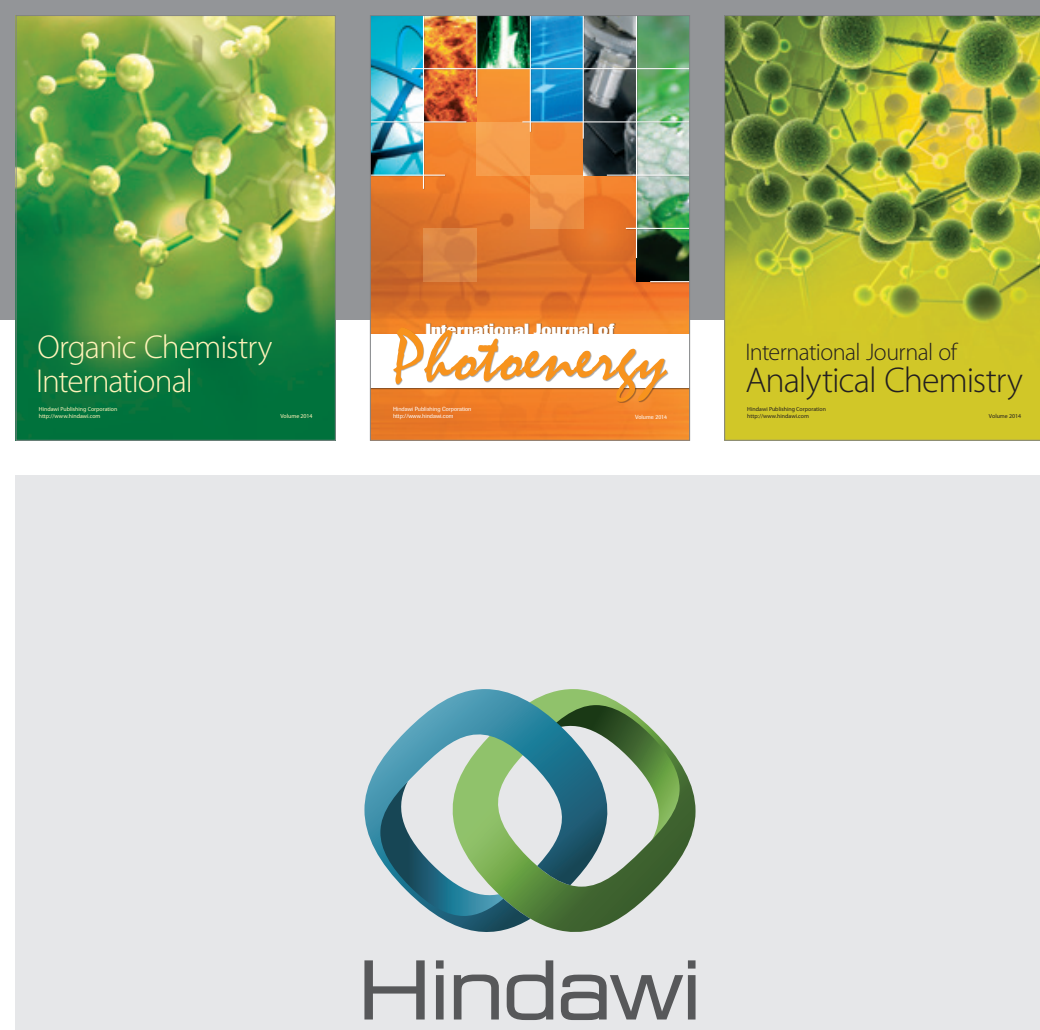

Submit your manuscripts at

http://www.hindawi.com
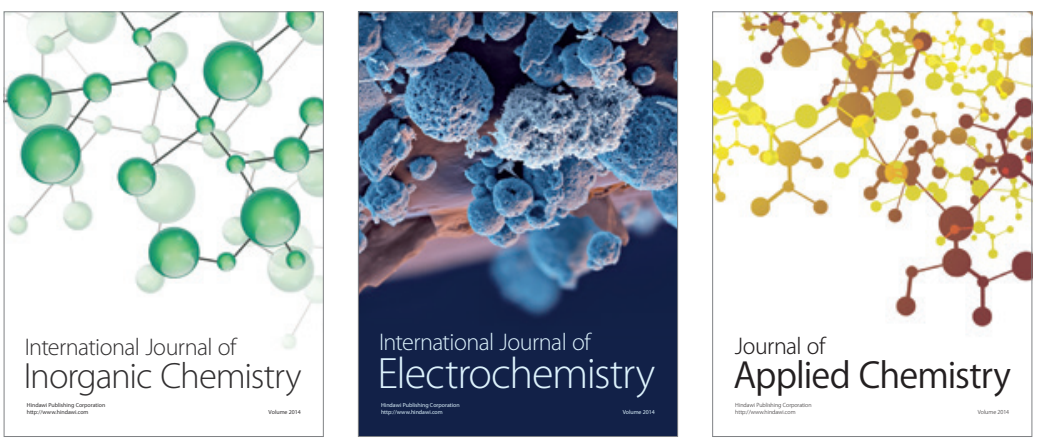

Journal of

Applied Chemistry
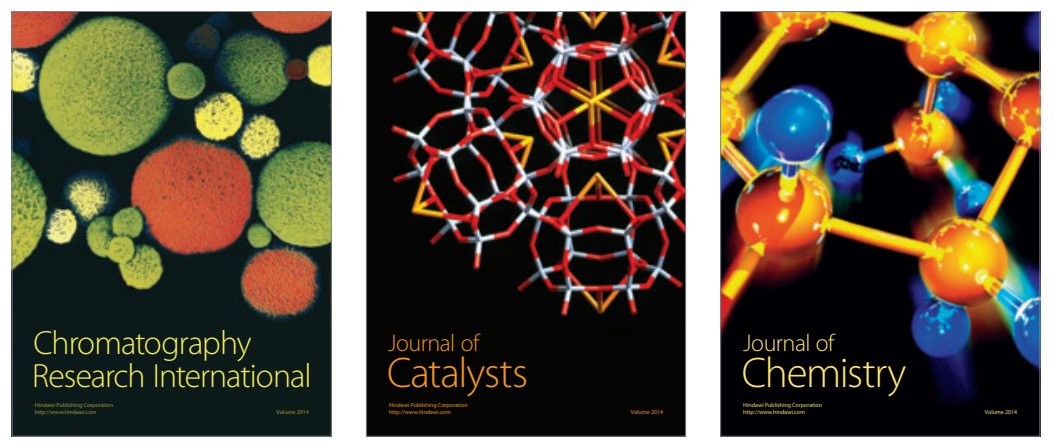
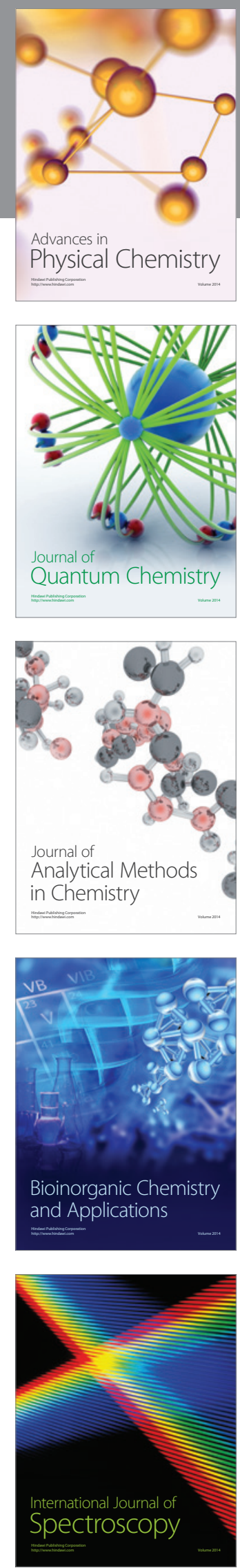